\title{
High performance dendrimer functionalized single-walled carbon nanotubes field effect transistor biosensor for protein detection
}

Rajesh, Vikash Sharma, Nitin K. Puri, Ashok Mulchandani, and Ravinder K. Kotnala

Citation: Appl. Phys. Lett. 109, 243504 (2016); doi: 10.1063/1.4972110

View online: http://dx.doi.org/10.1063/1.4972110

View Table of Contents: http://aip.scitation.org/toc/apl/109/24

Published by the American Institute of Physics

\section{Articles you may be interested in}

Substrate dependence of graphene reactivity towards hydrogenation

Appl. Phys. Lett. 109, 243103243103 (2016); 10.1063/1.4971385

Local conductance mapping of water-intercalated graphene on mica

Appl. Phys. Lett. 109, 241602241602 (2016); 10.1063/1.4972233

Manipulation of the electroluminescence of organic light-emitting diodes via fringe fields from patterned magnetic domains

Appl. Phys. Lett. 109, 243303243303 (2016); 10.1063/1.4971332

Influence of material impurities in the hole-blocking layer on the lifetime of organic light-emitting diodes

Appl. Phys. Lett. 109, 243302243302 (2016); 10.1063/1.4972055

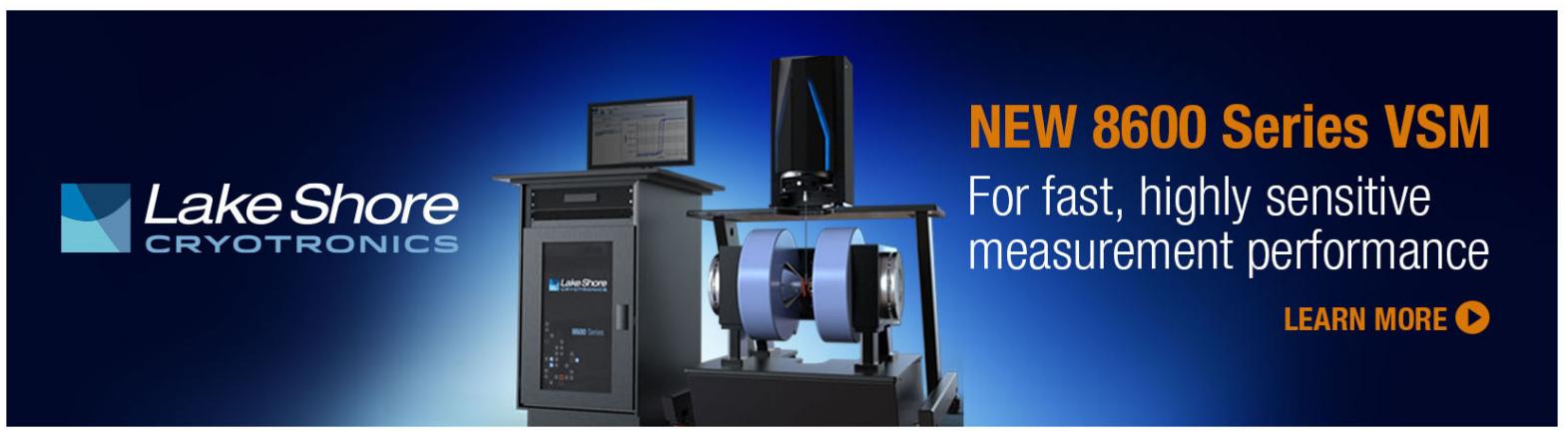




\title{
High performance dendrimer functionalized single-walled carbon nanotubes field effect transistor biosensor for protein detection
}

\author{
Rajesh, ${ }^{1, \text { a) }}$ Vikash Sharma, ${ }^{1,2}$ Nitin K. Puri, ${ }^{2}$ Ashok Mulchandani, ${ }^{3}$ and Ravinder K. Kotnala ${ }^{1}$ \\ ${ }^{1}$ CSIR-National Physical Laboratory, Dr. K.S. Krishnan Road, New Delhi 110012, India \\ ${ }^{2}$ Department of Applied Physics, Delhi Technological University, Bawana Road, Delhi 110042, India \\ ${ }^{3}$ Department of Chemical and Environmental Engineering, and Materials Science and Engineering Program, \\ University of California, Riverside, California 92521, USA
}

(Received 19 September 2016; accepted 30 November 2016; published online 16 December 2016)

\begin{abstract}
We report a single-walled carbon nanotube (SWNT) field-effect transistor (FET) functionalized with Polyamidoamine (PAMAM) dendrimer with 128 carboxyl groups as anchors for site specific biomolecular immobilization of protein antibody for C-reactive protein (CRP) detection. The FET device was characterized by scanning electron microscopy and current-gate voltage $\left(I-V_{g}\right)$ characteristic studies. A concentration-dependent decrease in the source-drain current was observed in the regime of clinical significance, with a detection limit of $\sim 85 \mathrm{pM}$ and a high sensitivity of $20 \%$ change in current $(\Delta I / I)$ per decade CRP concentration, showing SWNT being locally gated by the binding of CRP to antibody (anti-CRP) on the FET device. The low value of the dissociation constant $\left(K_{d}=0.31 \pm 0.13 \mu \mathrm{g} \mathrm{ml}^{-1}\right)$ indicated a high affinity of the device towards CRP analyte arising due to high anti-CRP loading with a better probe orientation on the 3-dimensional PAMAM structure. Published by AIP Publishing. [http://dx.doi.org/10.1063/1.4972110]
\end{abstract}

C-reactive protein (CRP) is an acute phase pentameric plasma protein, consisting of five identical $\sim 23 \mathrm{kDa}$ homologous subunits, which are non-covalently associated in a cyclic form. It is synthesized by the liver ${ }^{1}$ and widely accepted as a biomarker of cardiovascular diseases and inflammation. $^{2-4}$ The American Heart Association and the United States Centre for Disease Control have suggested three different levels of CRP concentrations for the evaluation of cardiovascular disease risk. ${ }^{5-8}$ CRP concentration less than $1 \mu \mathrm{g} \mathrm{ml}^{-1}$ represents a low risk state; concentration between 1 and $3 \mu \mathrm{g} \mathrm{ml}^{-1}$ is considered as an average risk state; and any CRP concentration above $3 \mu \mathrm{g} \mathrm{ml}^{-1}$ represents a high risk state. Currently, a number of CRP testing methods are available in clinical laboratories, such as nephlometric, ${ }^{9}$ turbidimetric, ${ }^{10}$ visual agglutination, ${ }^{11}$ and the enzymelinked immunosorbent assay (ELISA) kits. ${ }^{12}$ However, these methods are time-consuming, cost-ineffective, and are not readily applicable for the rapid point-of care analysis. ${ }^{13} \mathrm{CRP}$ quantification methods based on surface plasmon resonance (SPR), ${ }^{14}$ microfluidics, ${ }^{15}$ and electrochemistry ${ }^{16}$ have been developed during the past few years. However, majority of these techniques require labels, expensive equipment, and well-trained personnel. Therefore, a more straightforward methodology 17,18 with features of (i) high sensitivity and specificity; (ii) non-labeling method; (iii) rapid assays; and (iv) portable, disposable, and low cost is needed.

Single-walled carbon nanotubes (SWNT) have been extensively used as an effective transducer for the fabrication of highly sensitive field-effect transistor (FET) sensors because of their ultra high surface area to volume ratio, extreme electrical (conductance/resistance) sensitivity to absorption/perturbation of analyte molecule on its surface, and its facile surface modification possibilities. ${ }^{19}$ A major

\footnotetext{
${ }^{\text {a) }}$ Author to whom correspondence should be addressed. Electronic mail: rajesh_csir@yahoo.com
}

barrier for using SWNTs in nano and microelectronics applications has been associated with the difficulties in assembling the SWNTs precisely into small devices for scalable fabrication. This has been overcome by the use of random network of SWNTs for large area microelectronics devices, which exhibits the average properties of a large number of random individual SWNTs. In this work, we report a significant improvement in the performance of FET device fabricated with SWNTs networks between $3 \mu \mathrm{m}$ gap interdigitated 10 pairs of gold microelectrodes array on $\mathrm{SiO}_{2} / \mathrm{Si}$, for the quantitative detection of CRP in Phosphate Buffer Saline (PBS). Further, to facilitate and to improve the density of the biorecognition molecule, the SWNTs networks were functionalized with Polyamidoamine (PAMAM) dendrimer of generation 4.5 (G-4.5) with 128 carboxyl groups as anchors. The relatively high density of carboxyl group in G4.5 PAMAM ensures the incorporation of a large number of protein antibodies (anti-CRP) through carbodiimide coupling reaction on the SWNTs network surface for immunoreaction (antibody-antigen complex formation) with target protein CRP, producing a major decrease in the source-drain current, resulting to a high sensitivity Bio-SWNT-FET device. Therefore, the prime novelty of the manuscript is to highlight the significance of using G-4.5 PAMAM for the site specific biomolecular immobilization of protein antibody, anti-CRP, which provides a favorable microenvironment for the immunoreaction for antigen-antibody conjugation with a high output current response than earlier reported FET devices for CRP detection. ${ }^{20-22}$ This electronic immunosensor has additional advantages of being compatible with a low-cost and scalable fabrication procedure for the label-free detection of CRP biomarker.

The device is schematically presented in Fig. 1. The sensor fabrication, performance, and sensing mechanism were investigated by the measurement of the current as a function of the back-gate voltage $\left(I-V_{g}\right)$ characteristics. 


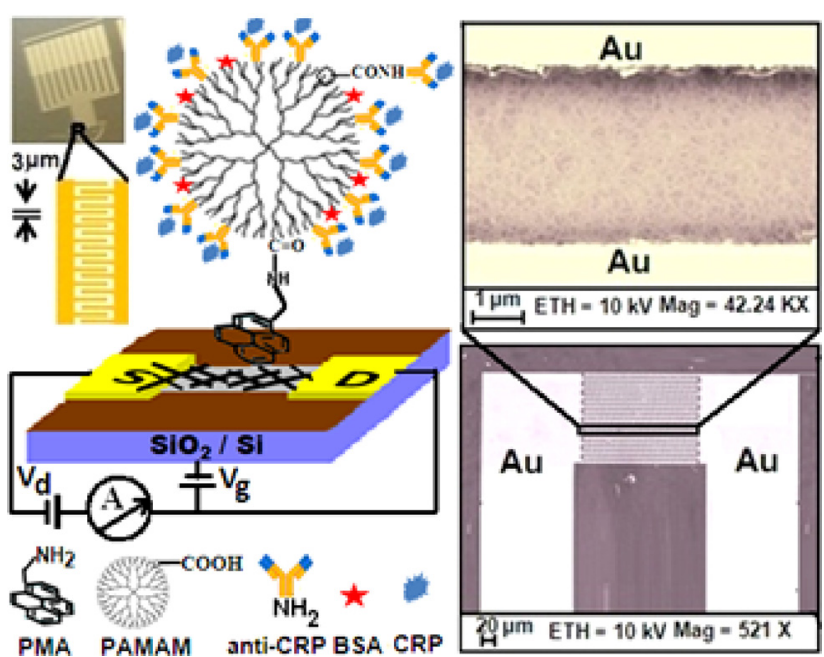

FIG. 1. Schematic illustration of the PAMAM modified SWNT-FET biosensor device with the SEM image of SWNT network between $3 \mu \mathrm{m}$ apart gold source-drain electrodes.

The SWNTs network was constructed on a 3aminopropyltriethoxy silane (APTES) treated $3 \mu \mathrm{m}$ gap interdigitated gold microelectrodes array on $\mathrm{SiO}_{2} / \mathrm{Si}$ (see details in the supplementary material). The scanning electron micrograph (FE-SEM, SUPRA $40 \mathrm{VP}$, Germany) image shows the high density of the SWNTs network $(\sim 65$ SWNTs $/ \mu \mathrm{m}^{2}$ ) between the electrodes (Fig. 1). The SWNTs network was first modified with the bifunctional linker, 1methyl pyrene amine, $\mathrm{PyMeNH}_{2}$, (PMA) by incubating it with $6 \mathrm{mM}$ PMA in methanol for $2 \mathrm{~h}$ at room temperature, washed with methanol, and dried under $\mathrm{N}_{2}$ flow. The $1 \mathrm{mM}$ aqueous PAMAM was treated with $0.1 \mathrm{M} \mathrm{N}$-(3-dimethyl aminopropyl)- $\mathrm{N}^{\prime}$-ethyl carbodiimide hydrochloride (EDC) and $0.05 \mathrm{M}$ N-hydroxy succinimide (NHS) for $3 \mathrm{~h}$ to activate the terminal carboxyl groups of PAMAM into the corresponding NHS-esters. The resultant activated PAMAM was dispensed over the PMA functionalized SWNTs network, where the part of the activated NHS-ester binds with the primary amine of PMA over the surface of the PMA/SWNTFET device. The AFM (Nanoscale 8, Bruker) image of the PAMAM modified SWNT-FET shows many randomly deposited globular shaped molecules over the SWNT surface (Fig. S1; see supplementary material). There are a few large irregular clusters perhaps caused by dendrimer aggregation. This is reflected by the height profiles of the globular particles on SWNT, which lie mostly about 6-8 nm with 1-2 nm diameter size SWNTs, which is consistent with $4.5 \mathrm{~nm}$ size diameter of the PAMAM dendrimer. Similar images have been obtained earlier for samples on mica substrates. ${ }^{23}$ The residual active NHS esters of the PAMAM dendrimer were bound to amines of anti-CRP during incubation of the PAMAM/PMA/SWNT-FET device with $100 \mu \mathrm{g} \mathrm{ml}^{-1}$ antiCRP protein, for a period of $3 \mathrm{~h}$, in phosphate buffer (PBS; $\mathrm{pH}$ 7.4) at $4{ }^{\circ} \mathrm{C}$ to obtain the desired anti-CRP-PAMAM/ PMA/SWNT-FET device.

We measured the $I-V_{g}$ characteristic of each device in an array of interdigitated 5 SWNT-FETs fabricated on a single chip, before and after successive steps of (1) SWNTs modification with PMA, (2) PAMAM functionalization of PMA/SWNT-FET device, and (3) bio-functionalization with
anti-CRP to confirm the formation of the bio-SWNT-FET devices (Fig. S2; see supplementary material). The Au source and drain electrodes were passivated with an 6-mercapto-1hexanol (MCH) self-assembled monolayer to avoid undesired Au surface contamination with anti-CRP protein during biofunctionalization. The electrical measurements were recorded using Agilent B 2902 A connected to a Micromanipulator model 450PM-B probe station by sweeping the gate voltage from $\pm 30 \mathrm{~V}$ at source-drain bias of $\left(V_{d s}\right)$ of $100 \mathrm{mV}$. The comparison of transistor characteristics of 5 devices revealed a high conduction to a comparatively denser SWNTs network, wherein source-drain current decreases strongly with increasing gate potential showing a p-type semiconducting behavior of the device (transport is dominated by holes) due to the electron withdrawal of the adsorbed oxygen molecules from the air. ${ }^{24}$ However, the devices does not show high on/off ratio, which may be due to the deleterious inter-CNT interaction in more densely packed arrays, as evident in the SEM image, which cause screening effects that decreases both the conductance and the on/off current ratio. ${ }^{25,26}$ The drain current continues to rise with increasing gate voltage without reaching a saturation, which may be due to the "source exhaustion," and the presence of carriers in the metal at energies within the CNT band gap causes tunneling to the channel through evanescent states in the CNT. ${ }^{27}$ The SWNT-FET devices show device to device $(n=5)$ hysteresis of $2 \pm 0.7 \mathrm{~V}$ between the forward and the reverse direction. This hysteresis is attributed to charge traps in bulk $\mathrm{SiO}_{2}$, oxygen-related defect trap sites near nanotubes, or at the $\mathrm{SiO}_{2} / \mathrm{Si}$ interface. ${ }^{28}$

The charge mobility $(\mu)$ of the devices was estimated using a standard expression for mobility, ${ }^{29} \mu=L g_{m} / W C V_{d s}$, where $L$ represents the length of the channel, $W$ is the width of the channel, $g_{m}$ is the transconductance of $d I / d V$ determined from each transfer characteristics in the linear regime, $V_{d s}$ is the source-drain voltage, $C$ is the approximate capacitance, $C=\varepsilon \varepsilon_{o} / L_{o x}$ where $\varepsilon$ is the dielectric permittivity of $\mathrm{SiO}_{2}(\varepsilon=3.9)$ and Lox is the thickness of the $\mathrm{SiO}_{2}$ layer. The value of $C$ for $300 \mathrm{~nm}$ thick $\mathrm{SiO}_{2}$ layer is $\sim 12 \mathrm{nF} \mathrm{cm}^{-2}$, which slightly overestimates the gate capacitance and correspondingly underestimates the carrier mobility due to the lower fill factor. ${ }^{30}$ The estimated mobility of the 5 SWNTFET devices was found to be in the range of $\sim 35$ to $\sim 65 \mathrm{~cm}^{2}$ $\mathrm{V}^{-1} \mathrm{~s}^{-1}$ (Fig. S2(a)). These variations in charge mobility are expected to depend on the device SWNTs network density as high density network renders a high mobility ${ }^{31}$ due to the availability of more paths for electron transport. After modification of SWNTs network with PMA, the threshold voltage $\left(V_{T h}\right)$ shifted towards the lower voltage direction with the decrease in the source-drain current and reduced charge mobility ranging between $\sim 7$ and $\sim 20 \mathrm{~cm}^{2} \mathrm{~V}^{-1} \mathrm{~s}^{-1}$ (Fig. S2(b)). This is attributed to the binding of PMA with SWNTs via $\pi-\pi$ linkage through pyrene moiety, where an increased scattering by the basic $\mathrm{NH}_{2}$ reduces the hole density in the SWNT channel. ${ }^{32}$ A further decrease in charge mobility was found $\left(\sim 3\right.$ to $\sim 16 \mathrm{~cm}^{2} \mathrm{~V}^{-1} \mathrm{~s}^{-1}$ ) with a major negative shift in the $V_{T h}$ due to the increased scattering on SWNTs by the NHS-ester activated PAMAM dendrimer in PAMAM modified SWNT-FET devices (Fig. S2(c)). The activated NHS-ester sites on the PAMAM/SWNT-FET device upon covalent binding with the protein antibody, anti-CRP, resulted 
in a further decrease in the charge mobility ( $\sim 2$ to $\sim 11 \mathrm{~cm}^{2}$ $\mathrm{V}^{-1} \mathrm{~s}^{-1}$ ) due to the charge transfer from the electron donating amine groups of arginine and lysine amino acids presents in the outer backbone chain of anti-CRP, where each amine group donates 0.04 electrons $^{33}$ to the nanotubes and hence reduces the hole density in the SWNT channel (Fig. S2(d)). A slight decrease in the charge mobility was obtained after blocking the uncovered non-specific sites on the SWNTs network and unbound free NHS-ester sites on PAMAM with a blocking reagent $(\mathrm{BR} ; 0.1 \%$ Tween-20 $+0.1 \% \mathrm{BSA}){ }^{26}$ The comparative electrical performance of an individual SWNTFET device (D1) with respective changes in both $V_{T h}$ and $\mu$ after the successive modifications, as discussed previously, is shown in Figs. 2(a) and 2(b). The substantive changes have been observed in charge density $(\rho)$ upon modification of the SWNT-FET device estimated by the equation ${ }^{29} \rho=C \Delta V_{T h} / e$ $L$, where $\Delta V_{T h}$ is the change in the threshold voltage. The SWNT device show a negative shift in $V_{T h}$ with a decrease in the hole density of $280 \times 10^{8} \mathrm{~cm}^{-1}$ for pyrene modified SWNT-FET with respect to bare SWNT-FET and $384 \times 10^{8} \mathrm{~cm}^{-1}$ for PAMAM dendrimer modified SWNTFET w.r.t. pyrene/SWNT-FET. This large decrease in the hole density on modification of SWNT-FET with PAMAM dendrimer reflected the high electron doping of SWNT from the negatively charged 128 carboxyl groups anchors, confirming the formation of PAMAM/SWNT-FET device. A further decrease of $36 \times 10^{8} \mathrm{~cm}^{-1}$ for anti-CRP-FET w.r.t. PAMAM modified SWNT-FET clarifies the scattering/charge transfer characteristics as discussed earlier. Figure 2(c) shows the output and transfer characteristics of the Bio-FET with the $I-V_{g}$ curve for $V_{d s}=0.1 \mathrm{~V}$ to $1.0 \mathrm{~V}$ and $I-V_{d s}$ curves (inset) measured for $V_{g}=-10 \mathrm{~V}$ to $-30 \mathrm{~V}$. The I- $V_{g}$ shows that the gate modulates the source-drain current by an increment of 2.5 times with a step of $V_{d s}=0.5 \mathrm{~V}$ and also the linear relationship between the $I-V_{d s}$ illustrates Ohmic contact between the SWNT and Au electrodes. In order to investigate the time period required for the completion of immunoreaction between the CRP analyte and anti-CRP on the channel surface of the Bio-FET device, we carried a real-time measurement of source-drain current upon exposure to $1.0 \mu \mathrm{g} \mathrm{ml}^{-1}$ target analyte CRP at $V_{d s}=0.1 \mathrm{~V}$ and $V_{g}=0 \mathrm{~V}$ (the inset of Fig. 2(d): upper-left side). A sharp decrease in the source-drain current with a gradual saturation at about $10 \mathrm{~min}$, indicated a near completion of the immunoreaction between the CRP and antiCRP and thus was used as immunoreaction incubation time. Rest, all the sensing operation has been done only at a fixed gate voltage of $V_{\mathrm{g}}=-30 \mathrm{~V}$.

To test the sensing performance of the device, a $5 \mu \mathrm{l}$ droplet of PBS containing CRP at a known concentration was dispensed on the sensor device and left to incubate for $10 \mathrm{~min}$ in humid environment, followed by washing with DI water, drying under gentle $\mathrm{N}_{2}$ flow, and the measurement of $I-V_{\mathrm{g}}$ characteristic was taken. A significant decrease in source-drain current was observed for both the PBS containing 1.0 and $10 \mu \mathrm{g} \mathrm{ml}^{-1} \mathrm{CRP}$ (Fig. 2(d)) and also for the $0.05 \mu \mathrm{g} \mathrm{ml}^{-1}$ CRP (inset of Fig. 2(d): lower-right side), indicating an effective immunoreaction taking place between anti-CRP and CRP analyte on the sensor device. This is probably due to increased carrier scattering and/or charge transfer from the electron donating amino groups of the target CRP analyte upon its binding to anti-CRP on the SWNT walls of FET device, as reported earlier. ${ }^{34}$ On the other hand, the exposure to pure PBS did not produce much change in the source-drain current (inset of Fig. 2(d): upper-right side).

Figure 3 depicts the plot of the percentage decrease in the ON state current at $V_{g}=-30 \mathrm{~V}$ and $V_{d s}=0.1 \mathrm{~V}$ normalized by the initial current $(\Delta I / I)$ versus CRP concentration in a logarithmic scale. Each data point in the plot was generated
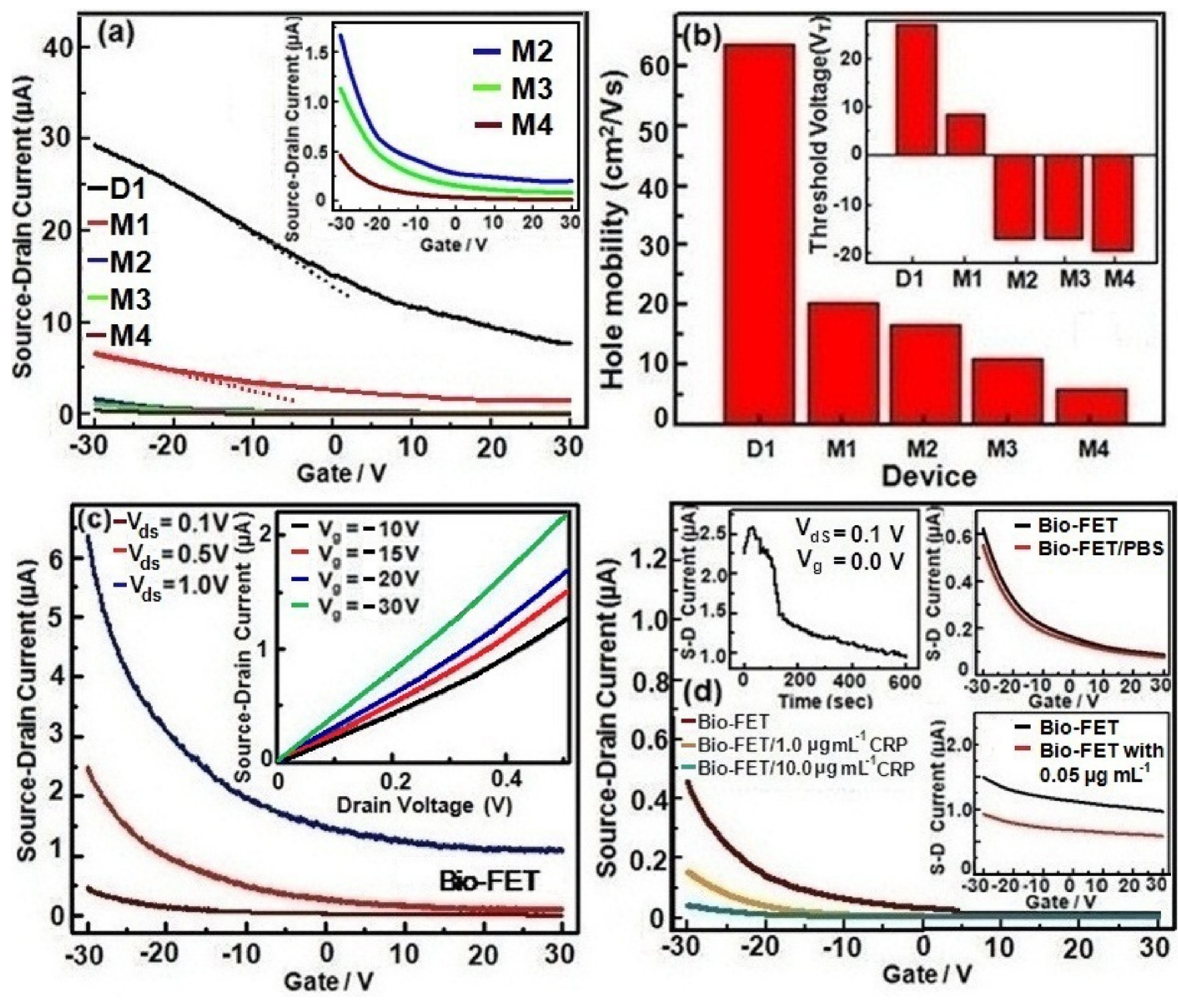

FIG. 2. (a) Current-gate voltage $\left(I-V_{\mathrm{g}}\right)$ characteristics of a representative SWNT-FET device, D1 (CNTFET) and after its successive chemical/ bio-functionalization, i.e., M1 (PMA/ CNT), M2 (PAMAM/PMA/CNT), M3 (anti-CRP-PAMAM/PMA/CNT) and M4 [(BR)anti-CRP-PAMAM/PMA/ CNT] Bio-FET; Inset shows the magnified view of the transfer characteristics. (b) Hole mobility of the device before and after each modification steps; The inset shows the threshold voltage of the corresponding device after each modification. (c) Current-gate voltage $\left(I-V_{\mathrm{g}}\right)$ characteristics of the Bio-FET at $V_{d s}=0.1$ to $1.0 \mathrm{~V}$; (d) $I-V_{\mathrm{g}}$ of device for $1.0 \mu \mathrm{g} \mathrm{ml}^{-1}$ and $10 \mu \mathrm{g} \mathrm{ml}^{-1} \mathrm{CRP}$; Insets show: response for $0.05 \mu \mathrm{g} \mathrm{ml}^{-1} \mathrm{CRP}$ (lower right side); the real-time sensor response to $1.0 \mu \mathrm{g} \mathrm{ml}^{-1} \mathrm{CRP}$ (upper left side), and sensor response to pure $10 \times 10^{-3} \mathrm{M}$ PBS (upper right side). 
by averaging the responses of 5 anti-CRP functionalized SWNT-FET devices tested against a solution with the same concentration of CRP; the error bar shown is the standard error of the mean. The sensor responses agree well with a model based on the Hill-Langmuir equation for equilibrium protein binding $^{35} \Delta I / I=\left[A\left(c / K_{d}\right)^{n} /\left\{1+\left(c / K_{d}\right)^{n}\right\}\right]+Z$, where $c$ is the CRP concentration, $A$ is the sensor response when all binding sites are occupied, $Z$ is an overall offset to account for the response to pure buffer, $K_{d}$ is the dissociation constant, and $n$ is the Hill coefficient describing cooperativity of binding. To construct the fit, the maximum response $A$ was constrained to a value $(A=77.3 \%)$ that was based on the observed response data and all other parameters, e.g., $K_{d}$, Z, and $n$ were allowed to vary. The best-fit parameters were $K_{d}=0.31 \pm 0.13 \mu \mathrm{g} \mathrm{ml}^{-1} \quad(2.6 \mathrm{nM}), \quad Z=8.1 \pm 3.4 \%$, and $n=0.55 \pm 0.03$. The low $K_{d}$ value is an indicative of strong anti-CRP binding to target CRP analyte on the sensor device, which in turn reflected the high anti-CRP loading on PAMAM dendrimer at the SWNT network FET device. A low value of $n=0.55$ corresponds to negative cooperativity, which may be due to inhibition of multiple CRP molecules binding to small aggregates or clusters of anti-CRP due to steric hindrance. The offset parameter $Z=8.1 \pm 3.4 \%$ was in good agreement with the measured responses of devices to pure buffer $(\Delta I / I=8.8 \pm 1.5 \%$; see Fig. 3$)$. The data presentation in Fig. 3 shows that the measured responses could be used to sufficiently differentiate between the pure buffer $(\Delta I / I$ $=8.8 \%$ ) and buffer containing CRP at a concentration of as low as $0.01 \mu \mathrm{g} \mathrm{ml}^{-1}$, or $\sim 85 \mathrm{pM}(\Delta I / I=18.5 \%)$. The changes in current response of the device are about $20 \%$ per decade concentration of the CRP (i.e., $2.0 \%$ current change in $\mu \mathrm{A}$ current signal for every $1.0 \%$ increase in CRP concentration). The sensitivity of the device for the same CRP concentration is much higher than various other material based FET devices. $^{20,21}$ A comparatively $\sim 10$ fold higher sensitivity obtained

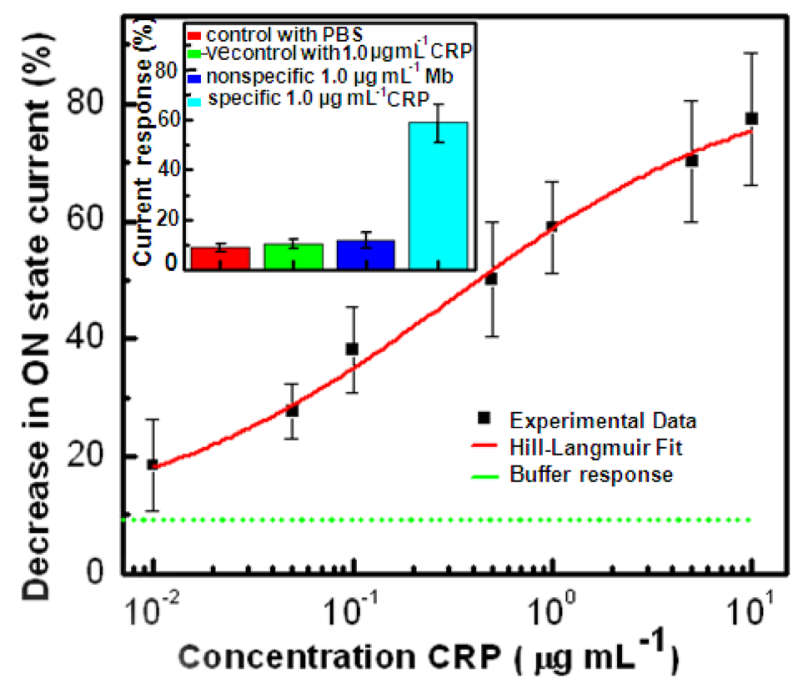

FIG. 3. The calibration curve of the Bio-FET device showing percentage decrease in the current response $(\Delta I / I)$ as a function of CRP concentration. The data are fit to a model based on the Hill-Langmuir equation (red curve); the inset shows the comparison of device responses for multiple control experiments. The Bio-FET device responses to $1.0 \mu \mathrm{g} \mathrm{ml}^{-1} \mathrm{CRP}$ (light blue bar), pure PBS buffer (red bar), to a non-specific protein marker $1.0 \mu \mathrm{g} \mathrm{ml}^{-1}$ myoglobin ( $\mathrm{Mb}$; dark blue bar), and a negative control response of the device without anti-CRP immobilization to $1.0 \mu \mathrm{g} \mathrm{ml}^{-1} \mathrm{CRP}$ (green bar). with the PAMAM based device with respect to the recently reported $^{22}$ bare SWNT-FET for the same concentration of CRP signifies the role using PAMAM for effective biomolecular immobilization of anti-CRP with enhanced loading and a better probe orientation due to its 3-dimensional polymeric structure on the SWNT device.

Multiple control experiments were performed to test the specificity of the device (inset to Fig. 3). A Bio-FET device was tested against buffer containing $1.0 \mu \mathrm{g} \mathrm{ml}^{-1}$ non-specific myoglobin $(\mathrm{Mb})$ protein, and the resulting response $(\Delta I / I$ $=11.6 \pm 2.8 \%$ ) was found to be nearly close to the device response to pure buffer $(\Delta I / I=8.8 \pm 1.5 \%)$ and much smaller than the response obtained for the same concentration of specific CRP $(\Delta I / I=58.9 \pm 7.8 \%)$, indicating towards an almost no interaction between the anti-CRP and Mb on the SWNTFET device (inset of Fig. 3). The response of the FET device without anti-CRP immobilization (negative control) to $1.0 \mu \mathrm{g}$ $\mathrm{ml}^{-1} \mathrm{CRP}$ analyte $(\Delta I / I=10.3 \pm 1.6 \%)$ was again found to be nearly comparable to the buffer response, indicating that the non-specific sites on the SWNT network were completely covered by the Tween-20 (blocking reagent), which prohibited the undesired reaction with the CRP analyte on the SWNT surface. This suggested a specific CRP analyte-antiCRP binding on the SWNT-FET device with a negligible contribution from nonspecific binding.

In conclusion, we have demonstrated a PAMAM dendrimer modified interdigitated SWNT-FET array device biofunctionalized with anti-CRP protein for the detection of CRP analyte. The device exhibited a concentration dependent response over a concentration range of $0.01 \mu \mathrm{g} \mathrm{ml}^{-1}$ to $10 \mu \mathrm{g} \mathrm{ml}^{-1}$ CRP in PBS that was in excellent quantitative agreement with a model based on the Hill-Langmuir equation of equilibrium thermodynamics. Multiple control experiments revealed that the anti-CRP immobilized on PAMAM retains its highly specific binding characteristics towards CRP analyte when attached to the SWNT network channel of the FET device. The high sensitivity of the PAMAM modified SWNT-FET device towards CRP than the previously reported ${ }^{22}$ sensor based on the bare SWNT device signifies the suitability of PAMAM for biomolecular immobilization with high loading and better probe orientation. These results of high antigen-antibody specificity with high sensitivity over the physiological range of CRP concentration and ease of use with a small sample volume make it superior to traditional immunoassay, suggesting that this device concept may be used as a portable tool to help diagnose disease after a further suitable optimization with real clinical (blood/human serum/plasma) samples.

See supplementary material for AFM images of PAMAM modified SWNT-FET device (Fig. S1) and a brief description of Current-gate voltage $(I-V \mathrm{~g})$ characteristics of the SWNT-FET devices after each successive modification steps (Fig. S2).

We are grateful to the Director, CSIR-National Physical Laboratory, New Delhi, India, for providing facilities. A.M. acknowledges the support of National Science Foundation Grant No. 1606181 and W. Ruel Johnson Chair in Environmental Engineering. We are thankful to Mr. V. V. 
Aggarwal and Bhasker Gahtori for providing the AFM and SEM images, respectively.

${ }^{1}$ M. B. Pepys and G. M. Hirschfield, J. Clin. Invest. 111, 1805 (2003).

${ }^{2}$ A. May and T. J. Wang, Expert Rev. Mol. Diagn. 7, 793 (2007).

${ }^{3}$ V. M. Miller, M. M. Redfield, and J. P. McConnell, Curr. Vasc. Pharmacol. 5, 15 (2007).

${ }^{4}$ N. D. Mygind, M. J. Harutyunyan, A. B. Mathiasen, R. S. Ripa, J. J. Thune, J. P. Gotze, J. S. Johansen, J. Kastrup, and C. T. Grp, Inflammation Res. 60, 281 (2011).

${ }^{5}$ I. Kushner and A. R. Sehgal, Arch. Intern. Med. 162, 867 (2002).

${ }^{6}$ L. R. Benzaquen, H. Yu, and N. Rifai, Crit. Rev. Clin. Lab. Sci. 39, 459 (2002).

${ }^{7}$ P. M. Ridker, Am. Heart Hosp. J. 2, 4 (2004).

${ }^{8}$ S. K. Mishra, R. Pasricha, A. M. Biradar, and Rajesh, Appl. Phys. Lett. 100, 053701 (2012).

${ }^{9}$ W. L. Roberts, R. Sedrick, L. Moulton, A. Spencer, and N. Rifai, Clin. Chem. 46, 461 (2000).

${ }^{10}$ W. L. Roberts, L. Moulton, T. C. Law, G. Farrow, M. Cooper-Anderson, J. Savory, and N. Rifai, Clin. Chem. 47, 418 (2001).

${ }^{11}$ N. P. Richards, T. S. Elliott, R. J. Powell, C. O' Callaghan, and P. Franklin, J. Clin. Pathol. 38, 464 (1985).

${ }^{12}$ E. M. Macy, T. E. Hayes, and R. P. Tracy, Clin. Chemistry 43, 52 (1997).

${ }^{13}$ T. A. Pearson, G. A. Mensah, Y. L. Hong, and S. C. Smith, Circulation 110, 543 (2004).

${ }^{14}$ W. P. Hu, H. Y. Hsu, A. Chiou, K. Y. Tsens, H. I. Lin, G. L. Chang, and S. J. Chen, Biosens. Bioelectron. 21, 1631 (2006).

${ }^{15}$ W. B. Lee, Y. H. Chen, H. I. Lin, S. C. Shiesh, and G. B. Lee, Sens. Actuators, B 157, 710 (2011).

${ }^{16} \mathrm{M}$. Buch and J. Rishpon, Electroanalysis 20, 2592 (2008).

${ }^{17}$ V. Vermeeren, L. Grieten, N. Vanden Bon, N. Bijnens, S. Wenmackers, S. D. Janssens, K. Haenen, P. Wagner, and L. Michiels, Sens. Actuators, B 157, 130 (2011).
${ }^{18}$ A. Qureshi, Y. Gurbuz, S. Kallempudi, and J. H. Niazi, Phys. Chem. Chem. Phys. 12, 9176 (2010).

${ }^{19}$ E. S. Snow, F. K. Perkins, E. J. Houser, S. C. Badescu, and T. L. Reinecke, Science 307, 1942 (2005).

${ }^{20}$ S. M. Kwon, G. B. Kang, Y. T. Kim, Y. H. Kim, and B. K. Ju, J. Nanosci. Nanotechnol. 11, 1511 (2011).

${ }^{21}$ Y. S. Sohn and Y. T. Kim, Electron. Lett. 44, 955 (2008).

${ }^{22}$ C. I. L. Justino, A. C. Freitas, J. P. Amaral, T. A. P. Rocha-Santos, S. Cardoso, and A. C. Duarte, Talanta 108, 165 (2013).

${ }^{23}$ D. A. Tomalia, S. Uppuluri, D. R. Swanson, and J. Li, Pure Appl. Chem. 72, 2343 (2000).

${ }^{24}$ A. Star, J. C. Gabriel, K. Bradley, and G. Gruner, Nano Lett. 3, 459 (2003).

${ }^{25}$ F. Léonard, Nanotechnology 17, 2381 (2006).

${ }^{26}$ A. Palaniappan, W. H. Goh, D. W. H. Fam, G. Rajaseger, C. E. Z. Chan, B. J. Hanson, S. M. Moochhala, S. G. Mhaisalkar, and B. Liedberg, Biosens. Bioelectron. 43, 143 (2013).

${ }^{27}$ V. Perebeinos, J. Tersoff, and W. Haensch, Phys. Rev. Lett. 111, 236802 (2013).

${ }^{28}$ W. Kim, A. Javey, O. Vermesh, Q. Wang, Y. Li, and H. Dai, Nano Lett. 3, 193 (2003).

${ }^{29}$ J. H. Lim, N. Phiboolsirichit, S. Mubeen, Y. Rheem, M. A. Deshusses, A. Mulchandani, and N. V. Myung, Electroanalysis 22, 99 (2010).

${ }^{30}$ E. S. Snow, P. M. Campbell, M. G. Ancona, and J. P. Novak, Appl. Phys. Lett. 86, 033105 (2005).

${ }^{31}$ Y. Zhou, A. Gaur, S. H. Hur, C. Kocabas, M. A. Meitl, M. Shim, and J. A. Rogers, Nano Lett. 4, 2031 (2004).

${ }^{32}$ Rajesh, Z. Gao, R. Vishnubhotla, P. Ducos, M. D. Serrano, J. Ping, M. K. Robinson, and A. T. C. Johnson, Adv. Mater. Interfaces 3, 1600124 (2016).

${ }^{33}$ K. Bradley, M. Briman, A. Star, and G. Gruner, Nano Lett. 4, 253 (2004).

${ }^{34}$ K. S. Vasu, K. Naresh, R. S. Bagul, N. Jayaraman, and A. K. Sood, Appl. Phys. Lett. 101, 053701 (2012).

${ }^{35}$ A. Hill, J. Physiol. 40, 4 (1910). 